\title{
Etude De L'arrière Effet Des Légumineuses Alimentaires Sur La Productivité Des Légumes : Cas De La Tomate (Solanum Lycopersicum), Cultivée Dans La Localité De Bouaké Au Centre De La Côte d'Ivoire
}

\author{
Noupé Diakaria Coulibaly \\ Christian Landry Ossey \\ André Gabazé Gadji \\ Mako François De Paul N'Gbesso \\ Lassina Fondio
}

CNRA (Centre National de Recherche Agronomique), Programme Cultures

Maraîchères et Protéagineuses, Côte d'Ivoire

Olivia Tiaplé Soro

INPRAT (Institut Privée d’Agriculture Tropicale), Côte d'Ivoire

\section{Doi:10.19044/esj.2021.v17n21p125}

Submitted: 30 March 2021

Accepted: 08 June 2021

Published: 30 June 2021
Copyright 2021 Author(s)

Under Creative Commons BY-NC-ND 4.0 OPEN ACCESS

Cite As:

Coulibaly N.D., Ossey C.L., Gadji A.G., N’Gbesso M.F.D.P., Fondio L. \& Soro O.T. (2021). Etude De L'arrière Effet Des Légumineuses Alimentaires Sur La Productivité Des Légumes : Cas De La Tomate (Solanum Lycopersicum), Cultivée Dans La Localité De Bouaké Au Centre De La Côte d'Ivoire. European Scientific Journal, ESJ, 17(21), 125.

https://doi.org/10.19044/esj.2021.v17n21p125

\section{Résumé}

Dans le but de proposer des méthodes agroécologiques pour améliorer la qualité et la quantité de production des légumes et aussi pour préserver l'environnement de l'utilisation abusive des intrants de synthèse, l'exploitation de l'arrière effet des légumineuses a été entreprise. Cette étude a donc pour objectif d'évaluer l'effet des légumineuses alimentaires sur la productivité de la tomate. Trois légumineuses alimentaires (arachide, niébé et soja) ont été mises en avant culture de la tomate à Bouaké localisée au centre de la Côte d'Ivoire. Après la récolte des légumineuses, un essai de fertilisation de la tomate a été mis en place selon un dispositif en blocs randomisés complets avec trois répétions. Les antécédents culturaux des légumineuses ont été utilisés comme source de fertilisation de la tomate. Deux témoins dont un 
sans aucune source de fertilisation et un autre témoin de référence qui est la pratique habituelle utilisant des engrais de fond (NPK) et de couverture (urée et sulfate de potasse). Les résultats obtenus ont montré que les paramètres de croissance, de développement végétatif et des stades phénologiques ont évolué indépendamment des sources de fertilisation. Par contre, la plupart des composantes du rendement ont été sources de variabilité entre les traitements. En effet, l'antécédent niébé a généré les rendements brut et net les plus élevés (7, 38 t/ha) par rapport autres traitements. Les traitements Témoin (T0), la référence (T1), les antécédentes culturales arachides et soja ont obtenu respectivement 2,$85 ; 5,31 ; 4,80$ et 2,35 t/ha. Le niébé pourrait être recommandé pour la culture de la tomate, vu la performance de son antécédent cultural.

Mots clés: Légumineuse, Tomate, Arrière Effet, Antécédent Cultural, Rendement, Côte d'Ivoire

\title{
Study Of The After-Effect Of Food Legumes On Vegetable Productivity: Case Of Tomato
}

\author{
Noupé Diakaria Coulibaly \\ Christian Landry Ossey \\ André Gabazé Gadji \\ Mako François De Paul N'Gbesso \\ Lassina Fondio
}

CNRA (Centre National de Recherche Agronomique), Programme Cultures

Maraîchères et Protéagineuses, Côte d'Ivoire

Olivia Tiaplé Soro

INPRAT (Institut Privée d’Agriculture Tropicale), Côte d'Ivoire

\begin{abstract}
In order to propose agroecological methods to improve the quality and quantity of vegetable production and also to preserve the environment from the abusive use of synthetic inputs, the exploitation of the after-effect of legumes was undertaken. The objective of this study is to evaluate the effect of food legumes on tomato productivity. Three food legumes (groundnut, cowpea and soybean) were put forward for tomato cultivation in Bouaké located in central Côte d'Ivoire. After harvesting the legumes, a tomato fertilization trial was set up in a complete randomized block design with three replications. The crop background of the legumes was used as a source of fertilizer for the tomato. Two controls, one without any source of fertilization and another reference control which is the usual practice using base (NPK)
\end{abstract}


and cover fertilizers (urea and sulphate of potash). The results obtained showed that the parameters of growth, vegetative development and phenological stages evolved independently of the sources of fertilization. However, most of the yield components showed variability between treatments. Indeed, the cowpea crop history generated the highest gross and net yields ( $7.38 \mathrm{t} / \mathrm{ha}$ ) compared to other treatments. The control (T0), reference (T1), groundnut and soybean treatments obtained 2.85, 5.31, 4.80 and 2.35 t/ha respectively. Cowpea could be recommended for tomato cultivation, given the performance of its crop history.

Keywords: Legume, Tomato, Back Effect, Crop History, Yield, Côte d'Ivoire

\section{Introduction}

L'agriculture représente le secteur clé de l'économie ivoirienne, occupant plus de $60 \%$ de la population active et fournissant en moyenne 30 à 35\% du produit intérieur brute, (MINAGRI, 2015). En Côte d'Ivoire, l'agriculture est constituée de grands composants dont l'agriculture d'exportation et vivrière. Bien que le succès de cette agriculture soit, principalement, basé sur les cultures de rente, le pays a de nos jours, opté pour une agriculture plus diversifiée. Cette diversification a eu pour conséquence le développement des cultures vivrières dont les cultures maraîchères (PNIA, 2017). Parmi ces cultures, la tomate a obtenu une place de choix auprès des producteurs et des consommateurs. Tant il est avéré qu'elle est riche en vitamines $C$, en provitamine $A$ et, surtout, en lycopène qui est l'antioxydant le plus actif des caroténoïdes alimentaires qui donne sa couleur rouge à la tomate (Rao et Agarwal, 2000).

D’une production de 27101 tonnes en 2006, la Côte d'Ivoire a réalisé une production de 34734 tonnes de tomate en 2013 (FAO, 2019). La production de tomate a, donc, considérablement augmenté. Pour maintenir cette production ou encore l'augmenté, les producteurs sont amenés à faire un maraîchage intensif sur des surfaces limitées. Dans la pratique actuelle, ils utilisent une importante quantité d'intrants agricoles, notamment, les insecticides, les fongicides et les engrais. Ces produits peuvent augmenter considérablement la production des cultures maraîchères. Ces intrants agricoles contiennent d'importantes quantités de substances chimiques, et ces substances ont trop souvent des effets néfastes sur la santé humaine et sur l'environnement. Ces substances peuvent s'accumuler dans les fruits sous forme de résidus. Et la consommation de tels fruits ou légumes peut être dangereuse pour la santé de l'homme. On pourrait, cependant, assister à une perturbation de l'équilibre des écosystèmes, à l'apparition des phénomènes de résistances des ravageurs aux pesticides utilisés et à la contamination des eaux et du sol. Face à ces menaces, il apparaît plus que nécessaire de trouver des 
solutions. Lesquels doivent viser à la préservation de la santé humaine et de l'environnement. Il faut, donc, mettre en place des techniques culturales qui réduiraient l'utilisation des produits chimiques.

C'est dans cette optique que la mise au point de méthodes agroécologiques pour la production de légumes a été envisagée. Il s’agit donc de méthodes qui tiennent compte de la qualité de production et de la préservation de l'environnement.

La présente étude a pour objectif d'étudier l'arrière effet des légumineuses alimentaires sur la productivité de la tomate.

\section{Matériel et méthodes}

\section{Zone d'étude}

L'étude a été réalisée à Bouaké, au centre de la Côte d'Ivoire, avec pour coordonnées géographiques, $7^{\circ} 46^{\prime}$ de latitude Nord, $5^{\circ} 06^{\prime}$ de longitude Ouest et $375 \mathrm{~m}$ d'altitude. La ville de Bouaké est située dans la zone de transition entre le climat forestier du Sud et le climat de la savane du Nord. Le climat est de type tropical humide, avec quatre saisons dont une grande saison sèche (novembre à février), une grande saison de pluies (mars à juin), une petite saison sèche (juillet à aout) et une petite saison de pluies (septembre à octobre).

\section{Matériel végétal}

Le matériel végétal utilisé dans cette expérimentation, provient de la collection du Programme Cultures Maraîchères et Protéagineuses du Centre National de Recherche Agronomique de Côte d'Ivoire. Trois (3) légumineuses alimentaires, notamment, l'arachide (Arachis hypogaea), le niébé (Vigna unguculata) et le soja (Glycine max) ont été retenues. Pour l'arachide, la variété 3-5A avec un cycle de 90 jours et un rendement potentiel de 1,5 t/ha a été préférée. Concernant le niébé, la variété KN1 dont le cycle de culture est de 90 jours et un rendement potentiel de 1,5 t/ha a été choisie. Quant au soja, la variété Canarana présentant un cycle de 90 jours et un rendement potentiel de 1,5 t/ha a, également, été choisi.

Le légume, étudié, a été la variété de tomate TM 44/14EW. Le cycle cultural de cette variété est de quatre-vingt-dix (90) jours avec un rendement potentiel de $30 \mathrm{t} / \mathrm{ha}$.

\section{Méthodes}

\section{Pratiques culturales}

Avant la mise en place de la tomate, une parcelle a été labourée et préparée pour recevoir les légumineuses (arachide, niébé et soja). En effet, l'essai a été conduit en deux (2) cycles de culture. Le premier cycle de culture a porté sur les légumineuses alimentaires. En fin de cycle, elles ont été 
récoltées. Après la récolte, les parcelles qui ont abrité les légumineuses ont été aménagées pour recevoir la culture de tomate.

Par ailleurs, une planche de $5 \mathrm{~m}^{2}$ a été confectionnée pour la mise en place de la pépinière. Après la confection de la planche, les semences de tomate ont été semées à raison de $10 \mathrm{~cm}$ entre les lignes et $5 \mathrm{~cm}$ entre les poquets. Cette pépinière a été ensuite recouverte par des feuilles de palmier à huile fraîches déposées à même le sol, et cela pendant les quatre (4) premiers jours. Après quatre (4) jours, une ombrière montée à une hauteur de $80 \mathrm{~cm}$ a été mise en place pour protéger les plants du soleil et des intempéries.

Vingt-un (21) jours après le semi en pépinière, les plants issus de la pépinière ont été repiqués sur des planches de $20 \mathrm{~m}^{2}$.

\section{Dispositif expérimental}

Sur le terrain, le dispositif expérimental utilisé pour la mise en place de l'essai a été des blocs randomisés complets. Les parcelles élémentaires ont été composées de quatre (4) billons de $5 \mathrm{~m}$ de longueur. Les billons étaient distants de $1 \mathrm{~m}$. La distance entre les plants sur le billon a été de 0,5 m. Les parcelles élémentaires ont été séparés de $1,5 \mathrm{~m}$. La superficie de chaque parcelle élémentaire était de $20 \mathrm{~m}^{2}$ (4 m x 5m).

Dans cette expérimentation, cinq (5) traitements ont été observés. Le témoin sans traitement (T0), la fertilisation de référence (FR), les arrières effets des légumineuses alimentaires ; en l'occurrence l'arrière effet arachide (AA), l'arrière effet niébé (AN) et l'arrière effet soja (AS). Chacun de ces traitements a été répété trois (3) fois. Chaque traitement est constitué d'une parcelle élémentaire de 4 billons. Cette expérimentation comprend au total 15 parcelles élémentaire ( 5 traitements $\times 3$ répétitions).

Pour la fertilisation de référence (FR), 300 kg/ha de NPK 102222 ont été apportés comme engrais de fond aux parcelles avant le repiquage. Ensuite, à la deuxième et à la cinquième semaine après le repiquage, un mélange de $100 \mathrm{~kg} / \mathrm{ha}$ d'urée et $200 \mathrm{~kg} / \mathrm{ha}$ de sulfate de potasse a été appliqué sur les mêmes parcelles. Les apports d'engrais de fond et de couverture ont été faits en les répandant sur les billons.

Les arrières effets des légumineuses alimentaires ont été utilisés comme fertilisants. Pour le niébé (AN) et le soja (AS), les tiges ont seulement été fauchées en laissant les racines dans le sol. En ce qui concerne l'arachide (AN), les racines ont été enfouies dans le sol après la récolte.

Pour toutes les légumineuses alimentaires, l'azote résiduel après culture issu de l'action symbiotique entre la légumineuse et les bactéries rhizobium sera exploité comme source d'apport d’éléments minéraux à la tomate. 
L'échantillonnage a porté sur les plants utiles des deux billons centraux ; les deux autres billons aux extrémités comportaient les plants de bordure.

\section{Observations et mesures}

Les observations et mesures ont porté sur différents paramètres de croissance et de rendement.

- Le développement végétatif et l'état sanitaire de 5 plants choisis au hasard à 30 et 60 jours après repiquage en attribuant des notes allant de 1 à 5 . ( 1 = développement végétatif /état sanitaire très mauvais ; $2=$ développement végétatif /état sanitaire mauvais ; 3 = développement végétatif /état sanitaire moyennement bon ; 4 = développement végétatif /état sanitaire bon ; $5=$ développement végétatif /état sanitaire très bon).

- Les stades phénologiques : le délai de floraison a été défini lorsque $50 \%$ des plants de la parcelle élémentaire ont fleuri. Le délai de première récolte représente le nombre de jours qui s'écoule entre le semis et le jour où la première récolte des fruits matures a été faite. Cette variable a permis de déterminer la précocité suivant les traitements. Le nombre de jours qui s'écoule entre la date de la première récolte et celle de la dernière récolte, a donné la durée de production en nombre de jours pour chaque traitement.

- les paramètres végétatifs prenant en compte la hauteur des plants, la canopée, le diamètre au collet, la longueur et la largeur de la feuille à la floraison sont mesurés sur cinq (5) plants échantillonnés par parcelle élémentaire. Pour la hauteur, les mesures ont été faites à l'aide d'un mètre ruban, en allant du collet au sommet du plant. Le mètre ruban a également servi à mesurer la canopée en déterminant la distance entre les deux extrémités du houppier. La longueur et la largeur de la feuille ont été mesurées en utilisant une règle graduée de $30 \mathrm{~cm}$. Un pied à coulisse a permis de déterminer le diamètre au collet du plant.

- La notation de la fermeté des fruits sains à la première récolte. Cette observation a été faite en choisissant au hasard 10 fruits sains par traitement dans chaque répétition. La notation de la fermeté des fruits s'est faite sur une échelle de 1 à 5 ( 1 = fruits complètement mous ; $2=$ fruits mous ; $3=$ fruits moyennement fermes ; 4 = fruits fermes ; 5 = fruits très fermes).

- Les composantes du rendement ont porté sur le nombre total de fruits, le nombre de fruits sains et le nombre de fruits avariés. A chaque récolte et pour chaque traitement, tous les fruits ont été comptés manuellement et pesés. Ensuite, les fruits sains et les fruits avariés (fruits perforés, éclatés, pourris, etc.) ont également été dénombrés et pesés. En fin de production, la somme des différents nombres et les poids totaux de fruits de chaque récolte ont été déterminés. Les poids totaux de fruits obtenus ont permis par extrapolation à l'hectare d'obtenir les rendements nets et potentiels en ( $\mathrm{t} / \mathrm{ha}$ ). 
Les rendements potentiels et nets ont été calculés selon la formule suivante :

$$
\begin{aligned}
& \text { Rendement potentiel }\left(\frac{\mathrm{t}}{\mathrm{ha}}\right) \\
& \qquad=\frac{\text { Poids total des fruits }(\mathrm{kg})}{\text { Surface pardellaire }\left(\mathrm{m}^{2}\right)} \times 10000 \frac{\llbracket\left(\mathbf{m} \rrbracket^{2}\right)}{1000}
\end{aligned}
$$

Le rendement net est obtenu en remplaçant le poids total des fruits par le poids total des fruits sains.

\section{Analyse statistique des données}

Les données collectées ont été analysées avec le logiciel Statistica version 7.1 où une analyse de variance (ANOVA) à un facteur a été réalisée pour l'étude de l'arrière effet des légumineuses alimentaires sur le rendement de la tomate (variété TM44). En cas de différence significative entre les moyennes, le test de Fisher au seuil de $5 \%(\alpha=0,05)$ a été effectué pour classer les moyennes en groupes homogènes.

\section{Résultats}

Développement végétatif et état sanitaire des plants de tomate de la variété TM44

Le tableau 1 présente le taux de reprise ; c'est-à-dire les plants ayant survécu 15 jours après repiquage (15 JAR), le développement végétatif (au $30^{\text {eme }}$ et $60^{\text {eme }} \mathrm{JAR}$ ) puis l'état sanitaire à 30 et $60 \mathrm{JAR}$ de la tomate de la variété TM44.

L'analyse de variance (ANOVA) appliquée au taux de reprise, aux paramètres du développement végétatif et à l'état sanitaire des plants a montré qu'il n'existe aucune différence $(P>0,05)$ entre les moyennes des traitements appliqués sur la variété de tomate TM44. Cependant, les valeurs numériques obtenues ont varié selon les traitements. En effet, le taux de reprise a été de 97,50 \%, chez le traitement de référence. La valeur du taux de reprise la plus petite $(89,15 \%)$ a été observée chez l'arrière effet arachide. Pour les développements végétatifs à 30 et $60 \mathrm{JAR}$, les valeurs les plus élevées ont été enregistrées respectivement chez l'arrière effet niébé (4) et tous les arrières effets des légumineuses (4 à 4,33). En ce qui concerne l'état sanitaire, l'arrière effet niébé a donné les valeurs les plus importantes (5) à 30 JAR. A 60 JAR, les valeurs les plus élevées (4) ont été notées chez les arrières effets arachide et soja. 
Tableau 1. Taux de reprise, paramètres de développement végétatif puis état sanitaire de la tomate TM44 en fonction des traitements

\begin{tabular}{|c|c|c|c|c|c|}
\hline Arrière effet & $\begin{array}{c}\text { TR 15JAR } \\
\mathbf{( \% )}\end{array}$ & $\begin{array}{c}\text { DV } \\
\text { 30JAR }\end{array}$ & DV 60JR & ES 30JAR & ES 60JAR \\
\hline T0 & $92,50 \pm 21,00^{\mathrm{a}}$ & $3,00 \pm 1,00^{\mathrm{a}}$ & $3,66 \pm 0,57^{\mathrm{a}}$ & $4,00 \pm 1,00^{\mathrm{a}}$ & $3,33 \pm 0,57^{\mathrm{a}}$ \\
T1 & $97,50 \pm 10,00^{\mathrm{a}}$ & $3,66 \pm 1,15^{\mathrm{a}}$ & $4,00 \pm 1,00^{\mathrm{a}}$ & $4,00 \pm 1,00^{\mathrm{a}}$ & $3,33 \pm 1,15^{\mathrm{a}}$ \\
Arachide & $89,15 \pm 15,20^{\mathrm{a}}$ & $3,00 \pm 0,00^{\mathrm{a}}$ & $4,00 \pm 0,00^{\mathrm{a}}$ & $4,00 \pm 0,00^{\mathrm{a}}$ & $4,00 \pm 0,00^{\mathrm{a}}$ \\
Niébé & $96,65 \pm 11,52^{\mathrm{a}}$ & $4,00 \pm 0,00^{\mathrm{a}}$ & $4,33 \pm 0,50^{\mathrm{a}}$ & $5,00 \pm 0,00^{\mathrm{a}}$ & $3,00 \pm 0,00^{\mathrm{a}}$ \\
Soja & $95,82 \pm 17,57^{\mathrm{a}}$ & $3,00 \pm 1,00^{\mathrm{a}}$ & $3,33 \pm 0,57^{\mathrm{a}}$ & $3,33 \pm 0,57^{\mathrm{a}}$ & $4,00 \pm 1,00^{\mathrm{a}}$ \\
\hline Moyenne & $94.32 \pm 15,06$ & $3,33 \pm 0,81$ & $3,86 \pm 0,63$ & $4,06 \pm 0,79$ & $3,53 \pm 0,74$ \\
\hline Probabilité & 0,06 & 0,45 & 0,41 & 0,13 & 0,39 \\
\hline CV (\%) & 4,50 & 24,32 & 17,21 & 19,45 & 20,96 \\
\hline
\end{tabular}

*Les chiffres dans la même colonne, affectés de la même lettre, ne diffèrent pas significativement au seuil de $5 \%$ (Test de Fisher)

TR 15JAR : Taux de reprise des plants 15 jours après repiquage ; $\mathbf{D V}$ : développement végétatif ; ES : état sanitaire

Le tableau 2 présente la hauteur des plants, la canopée, la surface foliaire et le diamètre au collet des plants de tomate de la variété TM44. L'analyse de ce tableau a indiqué que les valeurs des paramètres de croissance produites ont été statistiquement identiques au niveau de tous les traitements $(\mathrm{P}>0,05)$. Pour les descripteurs agromorphologiques tels que le diamètre au collet, l'indice de la vigueur végétative, la surface foliaire et la canopée, les valeurs les plus élevées (respectivement $0,80 \mathrm{~cm} ; 1,47 ; 40,44 \mathrm{~cm}^{2} ; 66,33 \mathrm{~cm}$ ) ont été observées chez l'arrière effet niébé. La fertilisation de référence (T1) en a généré les plus faibles valeurs (respectivement 0,64 cm; 1,25;27,60 cm² ; $53,33 \mathrm{~cm})$.

Tableau 2. Les valeurs de la hauteur des plants, canopée, surface foliaire et diamètre au collet de la tomate de la variété TM44

\begin{tabular}{|c|c|c|c|c|c|}
\hline Traitements & HP (cm) & DC (cm) & IVV & SF (cm $\mathbf{~}^{\mathbf{2}}$ & $\begin{array}{c}\text { Canopée } \\
(\mathbf{c m})\end{array}$ \\
\hline T0 & $59,33 \pm 8,57^{\mathrm{a}}$ & $0,78 \pm 0,09^{\mathrm{a}}$ & $1,45 \pm 0,03^{\mathrm{a}}$ & $27,87 \pm 5,88^{\mathrm{a}}$ & $56,06 \pm 17,60^{\mathrm{a}}$ \\
$\mathrm{T} 1$ & $54,73 \pm 5,46^{\mathrm{a}}$ & $0,64 \pm 0,02^{\mathrm{a}}$ & $1,25 \pm 0,09^{\mathrm{a}}$ & $27,60 \pm 10,44^{\mathrm{a}}$ & $53,33 \pm 12,39^{\mathrm{a}}$ \\
Arachide & $52,66 \pm 1,90^{\mathrm{a}}$ & $0,72 \pm 0,19^{\mathrm{a}}$ & $1,33 \pm 0,01^{\mathrm{a}}$ & $35,68 \pm 7,74^{\mathrm{a}}$ & $59,46 \pm 9,41^{\mathrm{a}}$ \\
Niébé & $59,20 \pm 4,35^{\mathrm{a}}$ & $0,80 \pm 0,04^{\mathrm{a}}$ & $1,47 \pm 0,05^{\mathrm{a}}$ & $40,44 \pm 7,92^{\mathrm{a}}$ & $66,33 \pm 5,03^{\mathrm{a}}$ \\
Soja & $57,60 \pm 5,04^{\mathrm{a}}$ & $0,72 \pm 0,10^{\mathrm{a}}$ & $1,37 \pm 0,07^{\mathrm{a}}$ & $30,44 \pm 7,12^{\mathrm{a}}$ & $59,13 \pm 6,70^{\mathrm{a}}$ \\
\hline Moyenne & $56,70 \pm 5,37$ & $0,73 \pm 0,10$ & $1,37 \pm 0,05$ & $32,40 \pm 8,46$ & $58,86 \pm 10,44$ \\
\hline Probabilité & 0,52 & 0,47 & 0,39 & 0,28 & 0,69 \\
\hline CV (\%) & 9,47 & 13,69 & 10,75 & 26,11 & 17,73 \\
\hline
\end{tabular}

*Les chiffres dans la même colonne, affectés de la même lettre, ne diffèrent pas significativement au seuil de $5 \%$ (Test de Fisher)

HP : hauteur des plants ; SF : surface foliaire ; DC : diamètre au collet

\section{Stades phénologiques des plants de tomate de la variété TM44}

Dans le tableau 3, sont indiquées les valeurs des stades phénologiques de la tomate TM44, à savoir, les dates de floraison, de la première récolte et 
de la dernière récolte. Les résultats obtenus ont montré que les valeurs des stades phénologiques, étudiés, ont été statistiquement identiques quel que soit la source de fertilisation. Mais numériquement, la fertilisation de référence a donné des valeurs précoces (42,33 JAR) du délai de floraison. Les valeurs tardives (48,33 JAR) du délai de floraison ont été obtenues par l'arrière effet soja. Concernant les délais de première et dernière récolte, la fertilisation de référence (T1) et l'arrière effet arachide ont occasionné les valeurs précoces (respectivement 63,00 JAR; 89,00 JAR). Les valeurs tardives (respectivement 67,67 JAR ; 93,67 JAR) ont été observées chez le témoin (T0).

Tableau 3. Date de floraison, date de $1^{\text {ère }}$ récolte et date de dernière récolte de la tomate de la variété TM44

\begin{tabular}{|l|l|l|l|}
\hline Traitements & DF (JAR) & D1ere R (JAR) & D D rec (JAR) \\
\hline T0 & $47,00 \pm 4,36^{\mathrm{a}}$ & $67,67 \pm 6,87^{\mathrm{a}}$ & $93,67 \pm 4,04^{\mathrm{a}}$ \\
\hline T1 & $42,33 \pm 6,43^{\mathrm{a}}$ & $63,00 \pm 6,40^{\mathrm{a}}$ & $89,00 \pm 0,00^{\mathrm{a}}$ \\
\hline Arachide & $45,00 \pm 2,65^{\mathrm{a}}$ & $63,00 \pm 6,40^{\mathrm{a}}$ & $89,00 \pm 0,00^{\mathrm{a}}$ \\
\hline Niébé & $43,00 \pm 2,65^{\mathrm{a}}$ & $65,33 \pm 6,63^{\mathrm{a}}$ & $91,33 \pm 4,04^{\mathrm{a}}$ \\
\hline Soja & $48,33 \pm 2,52^{\mathrm{a}}$ & $65,33 \pm 6,63^{\mathrm{a}}$ & $91,33 \pm 4,04^{\mathrm{a}}$ \\
\hline Moyenne & $45,13 \pm 4,14$ & $64,87 \pm 6,58$ & $90,87 \pm 3,20$ \\
\hline Probabilité & & & \\
& 0,36 & 0,38 & 0,38 \\
\hline CV (\%) & 9,17 & 10,15 & 3,52 \\
\hline
\end{tabular}

*Les chiffres dans la même colonne, affectés de la même lettre, ne diffèrent pas significativement au seuil de $5 \%$ (Test de Fisher)

DF : date de floraison en jours après repiquage (JAR) ; D1ereR : date de première récolte en JAR ; DDrec : date de dernière récolte en JAR

\section{Rendements et ses composantes des plants de tomate de la variété TM44}

Le tableau 4 montre le rendement et ses composantes de la tomate TM44, notamment, les poids totaux des fruits, des fruits avariés, des fruits par plant et le poids moyen des fruits. L'analyse de ce tableau a indiqué que deux (2) paramètres étudiés, à savoir, les poids des fruits avariés et le poids moyen des fruits ont de façon statistique, tous généré les mêmes valeurs quel que soit le traitement appliqué ; avec cependant, un poids de fruits avariés de plus de $3,97 \mathrm{~kg}$, chez la fertilisation de référence (T1) par rapport à l'arrière effet soja qui en a produit le plus faible $(1,10 \mathrm{~kg})$. Pour le poids moyen du fruit, les plus grandes $(22,62 \mathrm{~g})$ et plus petites $(16,64 \mathrm{~g})$ valeurs ont été observées respectivement chez l'arrière effet niébé et la fertilisation de référence (T1). Quant aux poids totaux des fruits, le poids de fruits sains et celui des fruits par plant, une différence $(\mathrm{P}=0,04$ et $\mathrm{P}=0,01)$ a été observée entre les moyennes obtenues avec les arrières effets des légumineuses. L'antécédent niébé a obtenu les plus grandes valeurs (respectivement $21,30 \mathrm{~kg} ; 17,79 \mathrm{~kg} ; 552,46$ g) de ces deux paramètres agromorphologiques. Les plus petits poids (respectivement 6, $03 \mathrm{~kg}$; 4,93 kg ; 157,34g) ont été générés par l'arrière effet soja. 
Tableau 4. Poids total des fruits, des fruits avariés, des fruits sains, des fruits par plant, et poids moyens du fruit de la tomate de la variété TM44.

\begin{tabular}{|c|c|c|c|c|c|}
\hline Traitements & P T Ft (kg) & P Fav (kg) & P Fts (kg) & P Ft/plt (g) & P moy Ft (g) \\
\hline T0 & $9,27 \pm 5,98^{\mathrm{bc}}$ & $3,29 \pm 2,56^{\mathrm{a}}$ & $5,98 \pm 2,70^{\mathrm{bc}}$ & $\begin{array}{c}246,05 \\
\pm 155,01^{\mathrm{c}}\end{array}$ & $17,06 \pm 6,67^{\mathrm{a}}$ \\
\hline T1 & $14,62 \pm 12,21^{\mathrm{ab}}$ & $3,97 \pm 4,38^{\mathrm{a}}$ & $10,65 \pm 3,26^{\mathrm{ab}}$ & $374,83 \pm 313,19^{\mathrm{b}}$ & $16,64 \pm 3,56^{\mathrm{a}}$ \\
\hline Arachide & $12,75 \pm 1,62^{\mathrm{b}}$ & $2,84 \pm 0,84^{\mathrm{a}}$ & $9,91 \pm 2,84^{\mathrm{b}}$ & $359,28 \pm 61,69^{\mathrm{b}}$ & $19,54 \pm 1,99^{\mathrm{a}}$ \\
\hline Niébé & $21,30 \pm 6,10^{\mathrm{a}}$ & $3,24 \pm 0,70^{\mathrm{a}}$ & $17,79 \pm 4,63^{\mathrm{a}}$ & $\begin{array}{c}552,46 \\
\pm 165,23^{\mathrm{a}}\end{array}$ & $22,62 \pm 4,24^{\mathrm{a}}$ \\
\hline Soja & $6,03 \pm 1,62^{\mathrm{c}}$ & $1,10 \pm 0,63^{\mathrm{a}}$ & $4,93 \pm 1,46^{\mathrm{c}}$ & $157,34 \pm 43,12^{\mathrm{d}}$ & $16,97 \pm 3,89^{\mathrm{a}}$ \\
\hline Moyenne & $12,79 \pm 7,82$ & $2,89 \pm 2,21$ & $9,9 \pm 7,26$ & $337,99 \pm 202,99$ & $18,57 \pm 4,36$ \\
\hline Probabilité & 0,04 & 0,64 & 0,04 & 0,01 & 0,43 \\
\hline CV (\%) & 61,14 & 76,47 & 54,83 & 60,05 & 23,47 \\
\hline
\end{tabular}

*Les chiffres dans la même colonne, affectés de la même lettre, ne diffèrent pas significativement au seuil de $5 \%$ (Test de Fisher)

PTFt : poids total des fruits ; PFav : poids des fruits avariés ; PFts : poids des fruits sains ; PFt/plt : poids des fruits par plant ; Pmoy Ft : poids moyen du fruit.

Le tableau 5 regroupe la fermeté des fruits, le nombre de récoltes, les nombres totaux de fruits et de fruits sains de la variété de tomate TM44. L'analyse du tableau a indiqué que la fermeté des fruits et le nombre de récoltes n’ont pas varié selon les traitements appliqués sur cette variété. Au plan numérique, les valeurs ont été plus importantes $(4,07)$ chez le témoin et l'arrière effet niébé en ce qui concerne la fermeté du fruit. Parmi les traitements utilisés, celui du soja a eu les plus faibles valeurs $(3,90)$. Pour le nombre de récoltes, les valeurs ont été importantes $(6,00)$ chez la fertilisation de référence et l'arrière effet arachide que celui du témoin qui en a donné les plus faibles $(5,33)$.

L'ANOVA des paramètres tels que les nombres totaux de fruits et de fruits sains a indiqué qu'il existe une différence significative entre les traitements. L'arrière effet niébé a donné les plus importantes valeurs (respectivement 928,33 ; 716,67) et celui du soja en a eu les plus petites (respectivement $381,67 ; 268,33)$.

Tableau 5. Fermeté des fruits, nombre de récoltes, nombre total de fruits, nombre de fruits sains de la tomate de la variété TM44.

\begin{tabular}{|c|c|c|c|c|}
\hline Traitements & Ferm ft & Nb rec & Nb Tft & Nb ft $s$ \\
\hline T0 & $4,07 \pm 0,51^{\mathrm{a}}$ & $5,33 \pm 0,58^{a}$ & $491,67 \pm 213,56^{d}$ & $\underset{c}{283,33 \pm 107,51}$ \\
\hline $\mathrm{T} 1$ & $3,93 \pm 0,35^{\mathrm{a}}$ & $6,00 \pm 0,00^{\mathrm{a}}$ & $836,67 \pm 628,83^{b}$ & $\begin{array}{c}536,67 \pm 355,61 \\
\text { b }\end{array}$ \\
\hline Arachide & $3,97 \pm 0,15^{\mathrm{a}}$ & $6,00 \pm 0,00^{\mathrm{a}}$ & $656,67 \pm 102,63^{c}$ & $465,00 \pm 74,67^{b}$ \\
\hline Niébé & $4,07 \pm 0,26^{\mathrm{a}}$ & $5,67 \pm 0,58^{a}$ & $928,33 \pm 108,67^{a}$ & $\underset{\text { a }}{716,67 \pm 101,16}$ \\
\hline Soja & $3,90 \pm 0,26^{\mathrm{a}}$ & $5,67 \pm 0,58^{\mathrm{a}}$ & $381,67 \pm 181,48^{e}$ & $268,33 \pm 85,20^{c}$ \\
\hline Moyenne & $3,99 \pm 0,31$ & $5,73 \pm 0,46$ & $659,00 \pm 340,14$ & $454,00 \pm 230,03$ \\
\hline
\end{tabular}




\begin{tabular}{|c|c|c|c|c|}
\hline Probabilité & 0,96 & 0,38 & 0,01 & 0,04 \\
\hline CV (\%) & 7,76 & 8,02 & 51,61 & 50,66 \\
\hline
\end{tabular}

*Les chiffres dans la même colonne, affectés de la même lettre, ne diffèrent pas significativement au seuil de $5 \%$ (Test de Fisher)

Ferm ft : fermeté des fruits ; Nb rec : nombre de récolte ; $\mathbf{N b} \mathbf{T} \mathbf{f t}$ : nombre total de fruits ;

$\mathbf{N b} \mathbf{f t} \mathbf{s}$ : nombre de fruits sains

Dans le tableau 6, sont consignés le nombre de fruits avariés, le nombre de fruits par plant, le rendement net et le rendement potentiel de la variété de tomate TM44. Les résultats obtenus ont montré qu'il existe une différence significative entre les moyennes des traitements appliqués en ce qui concerne tous ces descripteurs étudiés. Concernant le nombre de fruits par plant, les rendements potentiel et net, les valeurs les plus élevées (respectivement 24,06 ; 10,64 t/ha ; 7,38 t/ha) ont été enregistrées chez l'antécédent niébé ; alors que les plus faibles valeurs ont été produites par l'arrière effet soja (respectivement 9,$96 ; 3,01 \mathrm{t} / \mathrm{ha} ; 2,35 \mathrm{t} / \mathrm{ha}$ ). Par rapport au nombre de fruits avariés, l'arrière effet soja a donné le plus petit nombre de fruits $(105,00)$. Par contre, le plus grand nombre de fruits avariés $(300,00)$ a observé chez le traitement de référence $\mathrm{T} 1$.

Tableau 6. Nombre de fruits avariés, nombre de fruits par plant, rendements net et potentiel chez la variété de tomate TM44.

\begin{tabular}{|c|c|c|c|c|}
\hline Traitements & N Ft av & NFt $/$ plt & Rdt pot (t/ha) & Rdt net (t/ha) \\
\hline T0 & $208,33 \pm 107,28^{\mathrm{ab}}$ & $13,15 \pm 5,43^{\mathrm{b}}$ & $4,6^{\prime} \pm 2,99^{\mathrm{d}}$ & $2,85 \pm 1,66^{\mathrm{c}}$ \\
\hline T1 & $300,00 \pm 273,36^{\mathrm{a}}$ & $21,45 \pm 16,12^{\mathrm{a}}$ & $7,31 \pm 6,11^{\mathrm{b}}$ & $5,31 \pm 4,18^{\mathrm{b}}$ \\
\hline Arachide & $175,00 \pm 42,72^{\mathrm{ab}}$ & $18,50 \pm 3,64^{\mathrm{ab}}$ & $6,3 \_ \pm 8,11^{\mathrm{c}}$ & $4,80 \pm 1,24^{\mathrm{b}}$ \\
\hline Niébé & $211,67 \pm 16,07^{\mathrm{ab}}$ & $24,06 \pm 3,33^{\mathrm{a}}$ & $10,65 \pm 3,05^{\mathrm{a}}$ & $7,38 \pm 2,69^{\mathrm{a}}$ \\
\hline Soja & $105,00 \pm 85,29^{\mathrm{c}}$ & $9,96 \pm 4,80^{\mathrm{bc}}$ & $3,01 \pm 8,12^{\mathrm{e}}$ & $2,35 \pm 6,38^{\mathrm{c}}$ \\
\hline Moyenne & $200,00 \pm 133,83$ & $17,43 \pm 8,78$ & $6,40 \pm 3,91$ & $4,54 \pm 2,78$ \\
\hline Probabilité & 0,02 & 0,02 & 0,01 & 0,03 \\
\hline CV (\%) & 66,91 & 50,37 & 61,12 & 61,16 \\
\hline
\end{tabular}

*Les chiffres dans la même colonne, affectés de la même lettre, ne diffèrent pas significativement au seuil de $5 \%$ (test de Fisher).

$\mathbf{N}$ Fr av : nombre de fruits avariés ; $\mathbf{N}$ fr/plt : nombre de fruits par plant ; Rdt pot : rendement potentiel ;Rdt net : rendement net.

\section{Etude des corrélations entre les paramètres agromorphologiques}

Pour étudier les relations entre les paramètres agromorphologiques prises deux à deux, une matrice de corrélation dite matrice de Pearson a été générée. Seules les corrélations en gras ont été celles qui sont significatives au seuil de $5 \%$.

Les résultats consignés dans le tableau 7 ont montré que le développement végétatif à 30 jours après repiquage (JAR) est corrélé positivement avec le développement végétatif à $60 \mathrm{JAR}$, l'état sanitaire à 30 JAR, la hauteur des plants, la canopée, le nombre total de fruits, le nombre de 
fruit sains, le nombre de fruits avariés, le poids total de fruits, le poids de fruits avariés, le nombre de fruits par plant, le poids de fruits par plant, les rendements potentiel et net. Dans l'ensemble, les corrélations ont été fortes et positives ; sauf le délai de floraison qui a été négativement corrélé avec l'état végétatif à $30 \mathrm{JAR}$. En plus des paramètres tels que la surface foliaire et le poids moyen d'un fruit, ceux qui ont été corrélés à l'état végétatif $30 \mathrm{JAR}$, ont également été corrélés à l'état végétatif à 60 JAR. L'état sanitaire à 30 JAR a été lié positivement à la hauteur des plants, la canopée, la surface foliaire, le nombre total de fruits, de fruits sains, le nombre de fruits avariés, le poids total de fruits, le poids de fruits avariés, le nombre, le poids de fruits par plant, le poids moyen de fruits puis les rendements potentiel et net. La date de floraison quant à elle évolue négativement par rapport à l'état sanitaire $30 \mathrm{JAR}$. La canopée est positivement liée à la hauteur des plants et à la surface foliaire. La surface foliaire a donné une corrélation positive avec le nombre total de fruits, de fruits sains, le poids total de fruits, le nombre et poids de fruits par plant, le poids moyen de fruits et le rendement potentiel. Cependant, la corrélation a été négative avec le délai de floraison. Le délai de floraison a produit des corrélations négatives avec le rendement et ses composantes. Le nombre total de fruits a montré une forte liaison positive avec les nombres de fruits sains $(0,96)$ et avariés $(0,88)$, les poids totaux de fruits $(0,94)$, de fruits avariés $(0,81)$, de fruits par plant $(0,94)$, puis les rendements potentiel $(0,94)$ et net $(0,92)$. Le nombre de fruits sains a donné une forte corrélation positive avec le poids total de fruits $(0,97)$, le nombre de fruits par plant $(0,96)$, le poids de fruits par plant $(0,96)$, puis les rendements potentiel $(0,97)$ et net $(0,95)$. Cependant, la corrélation avec le nombre de fruits avariés et le poids de fruits avariés a été faible. Les paramètres qui ont évolué dans le même sens que le nombre de fruits avariés sont le poids total de fruits, le poids de fruits avariés, le nombre et le poids de fruits par plant puis les rendements potentiel et net. Par ailleurs, il a été observé que les paramètres tels que le poids de fruits avariés, le nombre de fruits par plant et les rendements potentiel et net ont été liés positivement au poids total de fruits. Le poids de fruits avariés a évolué positivement avec le nombre et le poids de fruits par plant et les rendements potentiel et net. Les paramètres tels que le poids de fruits par plant, les rendements potentiel et net ont fortement évolué et positivement avec le nombre de fruits par plant. Quant au poids de fruits par plant, il a été lié positivement au poids moyen de fruits puis aux rendements potentiel et net. Les rendements potentiel et net ont été liés positivement au poids moyen de fruit. Le rendement potentiel a augmenté positivement $(0,96)$ avec le rendement net. 
Tableau 7. Matrice de Pearson montrant les corrélations entre les paramètres agromorphologiques de la tomate TM44

\begin{tabular}{|c|c|c|c|c|c|c|c|c|c|c|c|c|c|c|c|c|c|c|c|c|c|c|c|c|}
\hline & $\begin{array}{c}\mathrm{NbP} \\
15 \\
\text { JAR }\end{array}$ & $\begin{array}{c}\text { Dev } \\
\text { Yeg } \\
30\end{array}$ & $\begin{array}{c}\text { Dev } \\
\text { Yeg } \\
60\end{array}$ & $\begin{array}{l}\text { ES } \\
30\end{array}$ & ES 60 & $\begin{array}{l}\mathrm{HP} \\
(\mathrm{cm})\end{array}$ & $\begin{array}{l}\text { Can } \\
(\mathrm{cm})\end{array}$ & $\begin{array}{c}\mathrm{SF} \\
\left(\mathrm{cm}^{2}\right)\end{array}$ & $\begin{array}{c}\mathrm{DC} \\
(\mathrm{cm})\end{array}$ & $\begin{array}{l}\text { DF } \\
\text { (j) }\end{array}$ & $\begin{array}{c}\text { D } \\
\text { lere } \\
\text { Rec } \\
\text { (i) }\end{array}$ & $\begin{array}{c}\text { D } \\
\text { Dres. } \\
\text { (j) }\end{array}$ & $\begin{array}{c}\text { Ferm } \\
\text { et }\end{array}$ & $\begin{array}{l}\mathrm{Nb} \\
\mathrm{NeC} .\end{array}$ & NbTft & $\underset{s}{\mathrm{NbE}}$ & $\begin{array}{l}\text { NbE } \\
\text { av }\end{array}$ & $\begin{array}{l}\text { PTFt } \\
(\mathrm{kg})\end{array}$ & $\begin{array}{c}\mathrm{Pft} \\
\text { av } \\
(\mathrm{kg})\end{array}$ & $\begin{array}{c}\mathrm{Nb} \\
\text { Ethl }\end{array}$ & $\begin{array}{c}\mathrm{Pft} / \mathrm{pl} \\
(\mathrm{g})\end{array}$ & $\begin{array}{c}\mathrm{P} \\
\mathrm{mgX} \\
\mathrm{ft} \\
(\mathrm{g})\end{array}$ & $\begin{array}{c}\text { Rdt } \\
\text { net } \\
(\mathrm{kg} / \mathrm{ha})\end{array}$ & PEs. \\
\hline NGP 15 JAR & 1,00 & & & & & & & & & & & & & & & & & & & & & & & \\
\hline Dev Yeg 30 & 0,38 & 1,00 & & & & & & & & & & & & & & & & & & & & & & \\
\hline Dev Yeg 60 & 0,23 & 0,77 & 1,00 & & & & & & & & & & & & & & & & & & & & & \\
\hline ES 30 & 0,17 & 0,84 & 0,86 & 1,00 & & & & & & & & & & & & & & & & & & & & \\
\hline ES 60 & 0,16 & 0,04 & 0,31 & 0,06 & 1,00 & & & & & & & & & & & & & & & & & & & \\
\hline $\mathrm{HP}(\mathrm{cm})$ & 0,16 & 0,62 & 0,34 & 0,64 & 0,16 & 1,00 & & & & & & & & & & & & & & & & & & \\
\hline $\begin{array}{l}\text { Canopte } \\
(\mathrm{cm})\end{array}$ & 0,19 & 0,59 & 0,59 & 0,71 & 0,18 & 0,68 & 1,00 & & & & & & & & & & & & & & & & & \\
\hline $\mathrm{SF}\left(\mathrm{cm}^{2}\right)$ & 0,13 & 0,51 & 0,73 & 0,69 & 0,29 & 0,38 & 0,85 & 1,00 & & & & & & & & & & & & & & & & \\
\hline $\mathrm{DC}(\mathrm{cm})$ & 0,08 & 0,21 & 0,16 & 0,32 & $-0,11$ & 0,41 & 0,50 & 0,51 & 1,00 & & & & & & & & & & & & & & & \\
\hline DF (j) & 0,32 & $-0,84$ & $-0,75$ & 0,72 & 0,07 & 0,35 & 0,62 & $-0,56$ & 0,18 & 1,00 & & & & & & & & & & & & & & \\
\hline $\begin{array}{l}\text { D lere Rec } \\
\text { (i) }\end{array}$ & 0,19 & $-0,25$ & $-0,11$ & 0,25 & $-0,24$ & 0,30 & 0,31 & $-0,16$ & 0,14 & 0,28 & 1,0 & & & & & & & & & & & & & \\
\hline D Drec (j) & 0,19 & $-0,25$ & $-0,11$ & 0,25 & $-0,24$ & 0,30 & 0,31 & $-0,16$ & 0,14 & 0,28 & 1,0 & 1,0 & & & & & & & & & & & & \\
\hline Eermo ft & 0,37 & 0,08 & 0,03 & 0,18 & $-0,03$ & 0,19 & 0,07 & 0,13 & 0,47 & 0,02 & 0,0 & 0,0 & 1,00 & & & & & & & & & & & \\
\hline $\mathrm{Nb}$ res. & 0,19 & 0,25 & 0,11 & 0,25 & 0,24 & 0,30 & 0,31 & 0,16 & 0,14 & 0,28 & $-1,0$ & $-1,0$ & 0,02 & 1,0 & & & & & & & & & & \\
\hline $\mathrm{NbJ \textrm {ft }}$ & 0,19 & 0,87 & 0,86 & 0,82 & 0,09 & 0,36 & 0,47 & 0,53 & 0,04 & 0,83 & $-0,2$ & $-0,2$ & 0,04 & 0,2 & 1,00 & & & & & & & & & \\
\hline $\mathrm{NbE} \mathrm{s}$ & 0,19 & 0,82 & 0,83 & 0,82 & $-0,01$ & 0,27 & 0,47 & 0,59 & 0,01 & 0,77 & $-0,2$ & $-0,2$ & 0,08 & 0,2 & 0,96 & 1,00 & & & & & & & & \\
\hline $\mathrm{NbE}$ av & 0,22 & 0,80 & 0,74 & 0,67 & 0,19 & 0,45 & 0,41 & 0,34 & 0,05 & 0,82 & $-0,2$ & $-0,2$ & $-0,03$ & 0,2 & 0,88 & 0,72 & 1,00 & & & & & & & \\
\hline PTEt (kg) & 0,20 & 0,79 & 0,86 & 0,85 & 0,02 & 0,33 & 0,51 & 0,61 & 0,05 & $0,7 i$ & $-0,1$ & $-0,1$ & 0,08 & 0,1 & 0,94 & 0,97 & 0,74 & 1,00 & & & & & & \\
\hline Pft av (kg) & 0,24 & 0,70 & 0,69 & 0,67 & 0,14 & 0,42 & 0,41 & 0,32 & an & 07 & $-0,1$ & $-0,1$ & $-0,13$ & 0,1 & 0,81 & 0,67 & 0,93 & 0,73 & 1,00 & & & & $\mathrm{G}$ & to \\
\hline $\mathrm{Nb}$ Ettpl & 0,10 & 0,85 & 0,84 & 0,81 & 0,10 & 0,35 & 0,46 & 0,52 & 0,06 & 0,81 & $-0,3$ & $-0,3$ & 0,07 & 0,3 & 1,00 & 0,96 & 0,87 & 0,94 & 0,80 & 1,00 & & & & \\
\hline $\mathrm{BAt} \mathrm{pl}(\mathrm{g})$ & 0,12 & 0,77 & 0,85 & 0,85 & 0,04 & 0,32 & 0,49 & 0,60 & 0,03 & 0,69 & $-0,2$ & $-0,2$ & 0,10 & 0,2 & 0,94 & 0,96 & 0,73 & 1,00 & 0,71 & 0,94 & 1,00 & & & \\
\hline P mox ft (8) & 0,20 & 0,28 & 0,54 & 0,57 & 0,11 & 0,22 & 0,48 & 0,55 & 0,10 & 0,15 & 0,0 & 0,0 & $-0,11$ & 0,0 & 0,40 & 0,50 & 0,18 & 0,64 & 0,33 & 0,40 & 0,65 & 1,00 & & \\
\hline Rdt pot (ha) & 0,20 & 0,79 & 0,86 & 0,85 & 0,02 & 0,33 & 0,51 & 0,61 & 0,05 & 0,71 & $-0,1$ & $-0,1$ & 0,08 & 0,1 & 0,94 & 0,97 & 0,74 & 1,00 & 0,73 & 0,94 & 1,00 & 0,64 & & \\
\hline $\begin{array}{l}\text { Rdt net } \\
\text { (kg ha) }\end{array}$ & 0,05 & 0,75 & 0,75 & 0,80 & 0,06 & 0,34 & 0,41 & 0,50 & 0,05 & 0,61 & $-0,3$ & $-0,3$ & 0,18 & 0,3 & 0,92 & 0,95 & 0,70 & 0,96 & 0,67 & 0,93 & 0,97 & 0,59 & 1,00 & \\
\hline PEs. & 0,01 & 0,17 & $-0,19$ & 0,11 & $-0,17$ & 0,13 & 0,26 & $-0,30$ & 0,11 & 0,09 & $-0,1$ & $-0,1$ & 0,05 & 0,1 & 0,07 & 0,14 & 0,09 & 0,08 & $0,0.8$ & 0,09 & 0,11 & 0,03 & 0,22 & 1,00 \\
\hline
\end{tabular}

\section{Discussion}

L’analyse des résultats obtenus à partir des différents paramètres de croissance et de développement végétatif, des stades phénologiques et des paramètres du rendement ont permis d'évaluer l'arrière effet de trois légumineuses alimentaire sur le comportement de la tomate (TM44).

En effet, l'analyse de variance en ce qui concerne les paramètres de croissance, de développement végétatif, l'état sanitaire, les stades phénologiques des plants n’a montré aucune variabilité entre les traitements. Les valeurs obtenues en étudiant les paramètres mentionnés ont évolué indépendamment des différentes sources de fertilisation utilisées. Il semble que ces traitements ont peu influencé les paramètres de croissance, de développement, l'état sanitaire et les stades phénologiques qui ont été mesurés. De ce point de vue, il est donc probable que les nutriments présents dans le sol avant la mise en place des légumineuses aient été en quantité suffisante pour la croissance et le développement des plants. Cela est d'autant vraisemblable si l'on se réfère au témoin sans traitement qui a eu la même 
performance que la fertilisation de référence et les arrière effets des légumineuses. Les résultats de cette étude ont confirmé ceux de Fondio et al. (2015). Selon eux, chez certaines variétés de tomate, les délais des stades phénologiques sont peu influencés par certains traitements tels que les apports d'engrais.

Les résultats obtenus en étudiant les paramètres du rendement ont indiqué que les descripteurs agromorphologiques tels que le nombre de récolte, la fermeté du fruit, le poids moyen du fruit et le poids des fruits avariés ont produit des valeurs statistiquement identiques quel que soit le traitement appliqué. Cependant, en prenant en compte les valeurs numériques, l'on note des poids moyen du fruit plus élevés chez l'arrière effet niébé suivi de celui de l'arachide. Les autres traitements (le témoin, la référence et l'arrière effet soja) en ont donné des valeurs inférieures. En se basant sur les performances du témoin sans traitement, il se pourrait que les éléments minéraux présents dans le sol avant l'application des traitements aient été en quantité suffisante. Avec une légère contribution des arrières effets niébé et arachide qui ont dû fournir une quantité supplémentaire de minéraux.

Par ailleurs, Benjamin (2019) en étudiant l'effet de trois types de composts et fertilisants chimiques sur la croissance et le rendement de la courgette a également noté qu'il n'y avait pas de différence significative entre les traitements pour le nombre de feuilles par plant. Par contre, une différence significative entre les traitements a été observée pour le poids moyen du fruit.

Parmi les paramètres du rendement et ses composantes qui ont été source de variabilité entre les traitements, l'on a noté : le nombre total de fruits, les nombres de fruits sains, de fruits avariés, de fruits par plant, les poids de fruits sains, et de fruits par plant puis les rendements potentiel et net. Ces descripteurs agromorphologiques ont donné les meilleures valeurs avec l'antécédent niébé. Les plus faibles valeurs ont été produites par l'arrière effet soja. Les rendements ont donc été plus élevés chez le traitement arrière effet niébé par rapport aux autres traitements. Ces résultats laissent à penser que le moment de la fructification a coïncidé avec la libération totale de l'azote contenu dans les nodules résiduels après la culture des légumineuses. Cela signifierait que la mise à disposition des éléments minéraux a été optimale après transformation de l'azote organique résiduel laissé par la légumineuse en fin de cycle de culture. Cela a permis aux plants du légume d'exprimer leur potentiel pour obtenir un rendement plus élevé. De ce point de vue, il semble que les éléments minéraux fournis par l'arrière effet du niébé ont été plus disponibles et ont également été en quantité plus élevée pour les plants de tomate en comparaison avec les autres traitements. En effet, le niébé, le soja et l'arachide sont des légumineuses qui abritent des bactéries rhizobium qui leur fournissent l'azote minéral à partir de l'azote atmosphérique. Cette symbiose est source de nutrition azotée pour les légumineuses, mais aussi 
permet d'enrichir la rhizosphère en azote. C'est donc dire qu'après la récolte, il subsiste une quantité résiduelle d'azote dans le sol. Et c'est cette quantité d'azote qui est utilisable par les cultures qui suivent celle des légumineuses. De ce point de vue, il est probable que la quantité d'azote laissée dans le sol par le niébé pour la tomate soit plus importante que les autres traitements. Les résultats de cette étude ont confirmé ceux de Konaté et al. (2012) qui ont noté que le niébé peut produire des nodules fixateurs plus importants soit 262 nodules. $\mathrm{m}^{-2}$ que chez le soja qui est de 199 nodules. $\mathrm{m}^{-2}$.

Selon Boubié (2002), les rhizobiums du niébé peuvent fixer jusqu'à 56 $\mathrm{kg}$ d'azote /ha. Or le traitement T1 a fourni $300 \mathrm{~kg}$ de NPK (10 22 22), soit 10

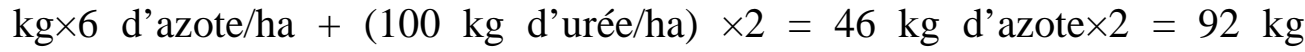
d'azote/ha; soit au total $102 \mathrm{~kg}$ d'azote/ha. L'on observe d'après ce qui précède, que T1 a finalement donné $102 \mathrm{~kg}$ d'azote/ha. T1 a donc apporté plus d'azote que le niébé ; et pourtant, les rendements ont été plus importants avec l'arrière effet niébé que T1. Cela pourrait s'expliquer par le fait que soit les pertes en azote dans le sol ont été élevées chez T1 soit que cette quantité d'azote ait été perçue comme un excès chez les plants de tomate ; ce qui a généré des rendements inférieurs à ceux du niébé.

Les résultats de cette étude ont également montré que les valeurs du rendement et ses composantes obtenues avec le témoin (parcelle sans traitement) ont été supérieures à ceux du soja. Cela signifierait que la quantité d'azote produite par la jachère a été importante que celle produite par l'arrière effet soja. Il est donc probable que les bactéries des rhizobiums du soja n'aient pas été efficientes au point de libérer assez d'azote dans la rhizosphère.

Mpika et al. (2015) puis Iqbal et al. (2015) ont, dans un autre contexte, montré que l'azote est essentiel au métabolisme de la plante. Son absorption suivie par son utilisation, conduit à la formation de protéines considérées comme les constituants essentiels de la formation de la matière vivante des plantes.

\section{Conclusion et perspectives}

L'étude de l'arrière effet des légumineuses alimentaires, sur la culture de tomate (TM44/EW), a montré que les traitements qui ont été appliqués sur les plants n’ont pas influencé les paramètres végétatifs et les stades phénologiques. En ce qui concerne le rendement et ses composantes, les poids de fruits avariés, le poids moyen de fruits, la fermeté des fruits et le nombre de récolte n’ont donné aucune différence significative entre les traitements. Par contre, les autres descripteurs tels que le nombre total de fruits, les nombres de fruits sains, de fruits avariés, de fruits par plant, les poids de fruits sains, avariés et de fruits par plant, les rendements potentiel et net ont été source de variabilité entre les traitements. Parmi les antécédents culturaux étudiés, c’est celui du niébé qui a généré les meilleurs résultats en termes de rendement. Les plus faibles résultats ont été enregistrés chez l'arrière effet 
soja. L'étude de l'arrière effet des légumineuses alimentaires en comparaison de la fertilisation minérale a permis d'observer que l'antécédent cultural du niébé pourrait être exploité pour la culture de tomate. Ce résultat pourrait donc être considéré comme un acquis majeur dans la recherche agronomique qu'il faut confirmer.

\section{References:}

1. Benjamin J. : Effet de trois types de composts et fertilisants chimiques sur la croissance et le rendement de la courgette (Cucurbita Pepo L.) dans des sols basaltiques et calcaires à la commune de Kenscoff, Haïti. Master de spécialisation en production intégrée et préservation des ressources naturelles en milieu urbain et péri-urbain. Faculté de Gembloux Agro-Bio Tech (GxABT), Liège Université, 63 pages, 2019.

2. Boubié V.B.: Rôle des légumineuses sur la fertilité des sols ferrugineux tropicaux des zones guinéenne et soudanienne du Burkina Faso. Thèse de Doctorat, Département des sols et de génie agroalimentaire, Faculté des Sciences de l'agriculture et de l’alimentation, Université Laval Québec, 197 pages, 2002.

3. FAO : La situation mondiale de l'alimentation et de l'agriculture 2019. Aller plus loin dans la réduction des pertes et gaspillages de denrées alimentaires. Rome. 203 pages, 2019.

4. Fondio L., N'zi J.C et Kobenan K. : Comportement agronomique et sanitaire de nouvelles lignées de piment dans le Sud de la Côte d'Ivoire. Journal of Applied Biosciences, 92: 8594-8609. DOI: http://dx.doi.org/10.4314/jab.v92i1.4, 2015.

5. Iqbal J., Mitchell D.C., Barker D.W., Miguez F., Sawyer J.E., Pantoja J. et Castellano

6. M. J.: Does Nitrogen Fertilizer Application Rate to Corn Affect Nitrous Oxide? (3)44: 771-779, 2015.

7. PNIA (Programme National D'investissement Agricole De Deuxième Génération 2017-2025): Ministère de l'agriculture et du développement rural de Côte d'Ivoire (MINADER). Abidjan, Rapport final Novembre 2017. 156 pages, 2017.

8. Rao A.V. et Agarwal S. Role of antioxidant lycopene in cancer and heart disease. The Journal of the American College of Nutrition, 19: 563-569, 2000.

9. Konaté Z., Gala B.T.J., Messoum F.G., Sekou A., Yao-Kouame A., Camara M. et Keli

10. Z.J. : Alternatives à la fertilisation minérale des sols en riziculture pluviale de plateau 
11. : apports des cultures du soja et du niébé dans la fertilité d'un ferralsol hyperdystrique au

12. Centre-ouest de la Côte d'Ivoire. Journal of Appllied Biosciences, 54 : 3859 - 3869, 2012.

13. MINAGRI : Politique d'orientation agricole en Côte d'Ivoire. Loi n ${ }^{\circ}$ 2015-537 du 20 juillet 2015 d'orientation agricole. Journal Officiel de la République de Côte D’Ivoire. 12 pages, 2015.

14. Mpika J., Attibayeba, Makoundou A. et Minani D. : Influence d'un apport fractionné en potassium et en azote sur la croissance et le rendement de trois variétés de tomate de la zone périurbaine de Brazzaville en République du Congo. Journal of Appllied Biosciences, 94 :8789 - 8800, 2015. 